\title{
The effect of consumption of natural non-dairy probiotics on human health
}

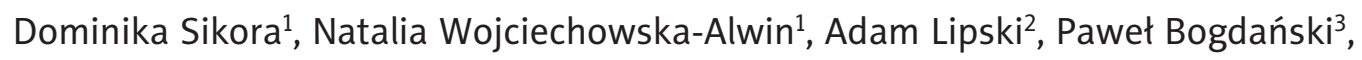 \\ Damian Skrypnik ${ }^{3}$
}

${ }^{1}$ Student Scientific Club of Clinical Dietetics, Department of Treatment of Obesity, Metabolic Disorders and Clinical Dietetics, Faculty of Medical Sciences, Poznan University of Medical Sciences, Poznan, Poland

${ }^{2}$ Department and Clinic of Urology and Oncological Urology, Poznan University of Medical Sciences, Poznan, Poland ${ }^{3}$ Department of Treatment of Obesity, Metabolic Disorders and Clinical Dietetics, Poznan University of Medical Sciences, Poznan, Poland

\begin{abstract}
Fermented food is a source of microorganisms. Numerous strains present in fermented products have probiotic characteristics. Probiotic strains can reverse dysbiosis due to the ability to modify gut microbiota. Regular consumption of fermented products may positively affect the composition and diversity of the intestinal microbiota. Numerous studies focus on the health effects of fermented dairy products. The range of non-dairy fermented products is large and includes, among others, vegetables, meat, fish, and eggs. The main ingredient and method of fermentation determine the presence and amount of bacterial strains. Lactic acid bacteria (LAB) present in sauerkraut, kimchi, and pickled cucumbers have a positive effect on the immune system activity, lipid metabolism, and anti-carcinogenic activity. The introduction of sauerkraut to the diet of irritable bowel syndrome (IBS) patients improved their quality of life. Soy sauce strains alleviate allergic rhinitis. Other fermented soy products show a beneficial effect on lipid profile or haematological parameters in animal models. Strains present in kombucha have not yet been tested for effects on consumer health. In summary, regular consumption of fermented non-dairy products can have a multidirectional impact on health improvement. However, more studies in this range, especially on appropriate daily intake of fermented food, are necessary to formulate a precise conclusion.
\end{abstract}

Key words: non-dairy probiotics, sauerkraut, kimchi, soy drink, tempeh.

\section{Introduction}

Gut microbiota is a diverse ecosystem consisting of over 1500 species of aerobic and anaerobic bacteria, fungi, and yeasts. The number of microorganisms is estimated at $10^{13}-10^{14}$ cells, and their total mass is above $2 \mathrm{~kg}$. The greatest number of microorganisms is found in the large intestine, due to the slower passage of this gut segment $[1,2]$. Microorganisms colonise the gut from the very early life period. Breastfeeding is particularly important in the development of gut microbiota. The composition of the microbiota varies individually, depending on many factors, e.g. labour mode, diet, and drugs [2,3].

The role of gut microbiota is not yet fully understood. Microorganisms living in the gut are responsible for metabolism and immunity. In addition, they perform trophic function on the intestinal epithelium [1]. Numerous studies indicate that quantitative and qualitative disturbances in the composition of microbiota called dysbiosis are associated with obesity, inflammatory bowel diseases, autoimmune disorders, cancers, or allergies $[1,4]$.

Probiotics are live microorganisms that, when administered in adequate amounts, confer a health benefit on the host [2]. They are mainly bacteria [1]. According to the suggestions of the World Health Organisation (WHO), to determine a strain as probiotic it must meet certain criteria, such as for safety and functionality. Probiotics do not include pathogenic and antibiotic resistant strains. Probiotics must survive in the digestive tract and have an immunomodulatory effect. In addition, they should have proven pro-health effects. Probiotic strains have to meet the requirements associated with the technology of production. These requirements include the probiotic strain's stability during all technological stages, e.g. storage and distribution [5]. 
Food containing more than one probiotic strain and at least $10^{6}$ active bacterial cells per millilitre of product is called a natural probiotic $[5,6]$. Many of these products are fermented, such as milk and dairy products, vegetables, fruits, legumes, meat, and eggs [7].

The positive impact of probiotics on human health has been confirmed in many studies. For example, Lactobacillus gasseri SBT2055 and Lactobacillus plantarum strain supplementation in obese patients decreases body mass and blood pressure. Probiotic strains such as Lactococcus lactis, Lactobacillus plantarum, Streptococcus cremoris, Lactobacillus casei, Streptococcus diacetylactis, Saccharomyces florentinusand, and Lactococcus remoris supplemented in lactose intolerant subjects improved the digestibility of lactose [5].

Numerous studies with natural probiotics focus on the health effects of fermented dairy products. This is due to the greater popularity of these products among consumers. The aim of this manuscript is to review current literature describing the effect of natural non-dairy probiotics on health.

\section{Non-dairy natural probiotics}

The benefits of the fermentation process are used not only in dairy products. Vegetables, fruits, cereals, legumes, meat, fish, and eggs can also undergo fermentation. The availability of different fermented products varies by region [7]. The assortment of non-dairy fermented foods is constantly expanding. This is mainly due to increased consumer interest [8]. Currently, a significant number of traditional and reginal fermented products are commercialised. An example is the currently readily available kimchi, which used to be mainly present in Korea and Asian countries. The most popular products in this range are pickled cucumbers, olives, and sauerkraut [9-11]. It has been shown that consumption of non-dairy fermented foods has a positive effect on the health of the consumer [10]. This is due to the presence of bacteria with probiotic properties [12].

\section{Cabbage-based fermented products}

Sauerkraut is one of the most popular non-dairy fermented products. World production is mainly focused on fermenting two types of cabbage. The Brassica oleracea variety is mainly found on the European and American markets. From the Brassica rapa L. subsp. pekinensis (so-called Chinese cabbage), popular in Asian countries, kimchi with a characteristic spicy taste is produced [11]. Lactic acid bacteria (LAB), which are naturally present in cabbage, are responsible for its fermentation. Production begins by adding edible salt $(\mathrm{NaCl})$. Its proper concentration causes the growth of lactic acid bacteria and inhibits the multiplication of putrefactive and pathogenic bacteria. Constant production conditions ensure the same composition of the microorganisms and their metabolites in the final product [13]. The microbiota of sauerkraut/ kimchi determines the taste, smell, and texture and is responsible for the fermentation process [14]. The average concentration of microorganisms in sauerkraut is from $10^{6}$ to $10^{9} \mathrm{cfu} / \mathrm{g}$ [10]. The starter microorganisms for sauerkraut and kimchi are, among others, Lactobacillus sakei, Lactobacillus plantarum, and Lactobacillus brevis. Fermentation usually occurs spontaneously [12].

Lactobacillus strains present in sauerkraut and kimchi have a positive effect on consumer health. Immunomodulatory effects increase in the body's immunity and decrease the cholesterol blood concentration after sauerkraut and kimchi consumption has been confirmed [14]. Lactobacillus plantarum is one of the main strains present in sauerkraut. Lactobacillus plantarum S2-5, S2-6, S4-1 are resistant to low gastric $\mathrm{pH}$, tolerate pancreatin and bile salts, and have the ability to adhere to intestinal cells, which fulfils the criteria of probiotic strain. Strain S4-1 decreases the concentration of low-density lipoprotein (LDL) cholesterol, triglycerides, and total cholesterol in blood of mice fed with milk enriched in a strain isolated from sauerkraut. High activity of beta-galactosidase has been demonstrated in strains S4-1 and S2-5. These strains are characterised by a higher degree of adhesion to the intestinal walls than the commercial probiotic Lactobacillus rhamnosus [15].

A pilot study conducted on a group of people with irritable bowel syndrome (IBS) showed a reduction in disease-related ailments measured by IBS-Symptom Severity Score (IBS-SSS) after the introduction of daily portion of $75 \mathrm{~g}$ of sauerkraut. In addition, the consumption of sauerkraut for six weeks increased the diversity of faecal bacteria. Positive effects were noted in the people consuming both pasteurised and unpasteurised sauerkraut [16].

The main ingredient of kimchi is Chinese cabbage. Depending on the region, the extras include garlic, ginger, and red pepper powder. In addition to spices, common ingredients of kimchi include radish, onion, leek, cucumber, and fermented seafood [17]. Fermentation is initiated through salinity. The process is analogous to cabbage pickling. In mature, commercially available kimchi, the dominant bacteria are Lactobacillus and Leuconostoc [18,19]. The strain of Lactobacillus acidophilus KFRI342 present in kimchi has a strong antiproliferative effect on cancer cells and a protective effect on damaged host cells. In addition, it has an immunostimulatory influence, causing a double increase in the synthesis of quantity reductase and stimulates the production of $\mathrm{NO}$ and IL-1a [17]. The LAB strains of kimchi (Lactobacillus plantarum, Leuconostoc mesenteroides, Lactobacillus brevis, Lactobacillus fermentum) inhibit mutation induced by 4-NQO (4-nitroquinoline-1oxide), MELQ (2-amino- 
3,4-dimethylimidaze [4,5-f] quinoline), and TRP-P2 (3-amino-1-methyl-5H-pyrido [4,3-b] indole), which are mutagenic compounds. Their effectiveness is the same as Lactobacillus acidophilus present in fermented dairy products. Lactobacillus plantarum extracted from kimchi and administered to mice resulted in increased synthesis of NO, tumour necrosis factor $\alpha$ (TNF- $\alpha$ ), and interleukin 6 (IL-6) in macrophage cells and a number of other effects positively affecting the immune system $[17,19]$. In mice given freeze-dried LAB, the anti-carcinogenic effect of Lactobacillus plantarum, Leuconostoc mesenteroides, and Lactobacillus case $i$ have been confirmed in the range of the development of lung cancer. In another study, $40 \mathrm{mg} / \mathrm{kg}$ /day of microorganism extracted from kimchi was administered to mice for 30 days. This intervention extended the survival time of the animals and inhibited the growth of the tumour [17]. Rats fed lyophilisate containing LAB isolated from kimchi showed significantly decrease blood levels of triglycerides and LDL cholesterol [18]. The effectiveness of kimchi in decreasing cholesterol levels is comparable to that of statins. The mechanism of action of LAB derived from kimchi is based on the reduction of cholesterol biosynthesis in the liver and increased lipid excretion in the faeces. Appropriate blood cholesterol level has a preventive effect on atherosclerosis development. Thus, regular consumption of kimchi may reduce the cardiovascular risk [20]. The administration of lactic acid bacterial lyophilizate collected from kimchi to rats led to weight loss and visceral fat content reduction. The Weissella koreensis OK1-6 isolated from kimchi inhibits the differentiation of 3T3-L1 cells, which are used as a research model to evaluate the development of adipocytes. The use of the Weissella koreensis strain as a starter of kimchi fermentation enhances its abilities to inhibit adipocytes growth. In addition, body mass reduction has been observed in humans consuming raw kimchi $[17,19]$.

The probiotic bacteria isolated from kimchi are characterised by antimicrobial, antifungal, and antiviral activity. These activities were maintained even after the exposure of LAB bacteria to proteolytic enzymes. Lactobacillus plantarum and Lactobacillus salivarius reduce the adhesion of Listeria monocytogenes and Escherichia coli to intestinal walls [17]. Moreover, consumption of kimchi causes changes in the intestinal microbiota due to the $\mathrm{LAB}$ content in the mature product. As a result of a twoweek supply of kimchi in the amount of $300 \mathrm{~g} /$ day, the abundance of Lactobacillus sp. and Leuconostoc sp. in the colon increased and the faecal pH decreased [17].

\section{Pickled cucumbers}

Lactic acid bacteria, mainly Lactobacillus plantarum, Lactobacillus pentosus, and Lactobacillus paraplantarum, are the main microorganisms responsible for the fermen- tation process of cucumbers [9,21]. LAB isolated from fermented cucumbers shows good ability to survive in the digestive tract [22]. Studies assessing consumption of strains of Lactobacillus spp. and Wiessella spp. isolated from cucumber showed significantly decrease blood cholesterol levels in study groups compared to controls. The decrease in cholesterol concentration is achieved by the influence of bacteria on the bile salts hydrolase activity. This leads to increased excretion of cholesterol with the bile. In addition, studied strains have shown activity against pathogenic yeast [23]. Lactobacillus plantarum in pickled cucumbers contribute to a reduction of inflammatory processes observed in Crohn's disease (CD) and ulcerative colitis (UC). This strain reduces the blood level of total and LDL cholesterol [24].

\section{Fermented soy-based products}

Soybean is a plant of the Fabaceae family, characterised by a high content of protein and low content of saturated fatty acids (SFA). Asian countries have the most experience in the cultivation and consumption of soy and its products. Currently, there is growing interest in soy and its preparations in Europe and in the USA, especially among consumer groups using diets that eliminate animal proteins. A correlation was observed between regular consumption of soybean and soy products and a lower incidence of cardiovascular diseases. Aspergillus fungus and the Bacillus subtilis strain are usually used in soy fermentation [25]. Soy-based preparations include tofu (soy curd), miso, soy drink, soy sauce, tempeh, textured soy protein, natto, sufu, and douche [12,25].

Soy drink is obtained by soaking soya beans in water. Some soy drinks are fermented during production by adding a probiotic starter. The main strains in fermented soy drinks are Lactobacillus acidophilus and Bifidobacterium. Laboratory studies on rats administered fermented soy drink showed its health-promoting effects. After 10 days of intervention, blood cholesterol levels were reduced, and after 40 days reduction in triglyceride blood content was registered as an effect of probiotic bacteria present in soy drink [26]. Studies performed on animal and human models have demonstrated an improvement in the lipid profile after supplementation of fermented soy drink based on the Enterococcus faecium strain. In rabbits fed with this soy drink for 15 days a decrease in total cholesterol level by $18.4 \%$ and increase in HDL cholesterol by $17.8 \%$ was demonstrated [27]. The use of a soy drink enriched with Lactobacillus plantarum has contributed to a significant improvement in haematological parameters such as haemoglobin level, and erythrocyte and leukocyte counts. In addition, the blood level of immunoglobulin (Ig) A antibodies was increased [26,28]. Other studies on mice fed lyophilisate of fermented soy drink showed an 
improvement in femoral microstructure. The drink used in the study was produced using the starter Lactobacillus paracasei or Lactobacillus plantarum bacteria [27]. Obese rats consuming the extract from fermented soy drink containing mainly Lactobacillus paracasei reduced body mass despite a high-fat diet consumption. The use of the extract containing Lactobacillus paracasei decreased the formation of atherosclerotic plaque, reduced the diameters of adipocytes, and decreased the absorption of lipids from the intestinal lumen [29]. A fermented beverage based on the Bifidobacterium breve strain presented anti-mutagenic activity. The use of lyophilisate of this drink in rodents with breast cancer reduced the number of tumours [27].

Tempeh is an Indonesian traditional protein product enriched with vitamin B12. It is made by boiling soy husk and fermentation by the addition of a starter containing a mixture of fungi Rhizopus. Instead of fungi, a starter with a mixture of Enterococcus faecium, Citrobacter freundii, and Klebsiella pneumonia is also used. Citrobacter freundii and Klebsiella pneumonias strains are responsible for the presence of vitamin B12 in the final product. Enterococcus faecium inhibits the growth of pathogenic bacteria such as Listeria monocytogenes and Escherichia coli [27]. In Sprague-Dawley rats fed tempeh for 28 days bacterial changes in faeces were observed. The abundance of bacteria Clostridium leptum, Bacteroides fragilis, Bacteroidetes, and Firmicutes increased. In the control group of animals fed non-fermented soy, no changes in faeces were found [30]. In a human study on volunteers, significant changes in faeces microbiota after consumption of tempeh were registered - the number of Akkermansia muciniphila increased. The study presented a possibility to modify the intestinal microbiota due to the consumption of tempeh [12].

Another soy-based fermented product is soy sauce. Soy sauce is an Asian spice with characteristic umami taste. The basis of soy sauce is boiled soy, to which roasted wheat flour and salt solution is added. Depending on the origin, the proportions of soy to flour varies. Fermentation is dominated by lactic acid bacteria and filamentous fungi such as Aspergillus oryzae and yeast [27]. The bacteria responsible for fermentation are also Bacillus, Klebsiella, Cladosporium, and Shimwellia [11]. Tetragenococcus halophilus isolated from soy sauce presents immunomodulatory effects. In patients who received supplementation of the Tetragenococcus halophilus strain isolated from soy sauce, a reduction in the symptoms associated with allergic rhinitis, a decrease in the serum concentration of $\operatorname{IgE}$ class antibodies, and a significant change in the Th1/Th2 cell ratio was demonstrated [27].

\section{Kombucha}

Kombucha is a product of black or green tea fermentation with sugar due to the addition of symbiotic bacte- ria and yeasts. The starter mixture of yeasts and bacteria is defined as Symbiotic Culture Of Bacteria and Yeast (SCOBY). SCOBY is a broad term for acetic acid bacteria (AAB) and yeasts. $\mathrm{AAB}$ include Acetobacter xylinum, Acetobacter aceti, and Gluconobacter oxydans [12,31,32]. Lactic acid bacteria also take part in the fermentation process, with higher concentration if green tea is used in production [33]. Kombucha is a low-alcohol drink - the alcohol content is $0.7 \%$ to $1.3 \%$. The popularity of kombucha in Western countries is increasing. There are still very few studies regarding the impact of kombucha on human health. Nevertheless, kombucha is referred to as a functional food due to the high content of polyphenols, flavonoids, and probiotic microorganisms [12,31].

\section{Conclusions}

The consumption of non-dairy fermented products beneficially affects many aspects of human health. It has been confirmed that its consumption changes the microbiota of the human gut. Studies have shown a relationship between the supplementation of specific probiotic strains, body mass reduction, and lipid profile improvement. It seems beneficial to introduce people with metabolic syndrome components and some other diseases to a diet rich in non-diary fermented products. Probiotic strains present in this group of foods modify the immune system and reduce ailments in people with IBS. Regular consumption of non-dairy fermented products certainly has a positive effect on maintaining the balance of the intestinal microbiota. Further research in this topic is needed to draw a precise conclusion, especially to determine the dose effect of non-diary probiotic food on human health.

\section{Disclosure}

The authors report no conflict of interest.

\section{References}

1. Krakowiak O, Nowak R. Mikroflora przewodu pokarmowego człowieka - znaczenie, rozwój, modyfikacje. Postępy Fitoter 2015; 16: 193-200.

2. Skrypnik K, Suliburska J. Association between the gut microbiota and mineral metabolism: Gut microbiota and mineral metabolism. J Sci Food Agric 2018; 98: 2449-2460.

3. Jenmalm MC. The mother-offspring dyad: microbial transmission, immune interactions and allergy development. J Intern Med 2017; 282: 484-495.

4. Gregorczyk-Maślanka K, Kurzawa R. Mikrobiota organizmu ludzkiego i jej wpływ na homeostazę immunologiczną - część I. Alerg Astma Immunol 2016; 21: 146-150.

5. Markowiak P, Śliżewska K. Effects of probiotics, prebiotics, and synbiotics on human health. Nutrients 2017; 9. pii: E1021.

6. Mojka K. Probiotyki, prebiotyki i synbiotyki - charakterystyka i funkcje. Probl Hig Epidemiol 2014; 95: 541-549.

7. Campbell-Platt G. Fermented foods - a world perspective. Food Res Int 1994; 27: 253-257. 
8. Terefe NS. Food fermentation. In: Reference module in food science. Elsevier 2016; doi: 10.1016/B978-0-08-100596-5.03420-X.

9. Harris LJ. The microbiology of vegetable fermentations. In: Microbiology of fermented foods. Wood BJB (ed.). Springer, Boston MA 1997; 45-68.

10. Marco ML, Heeney D, Binda S, et al. Health benefits of fermented foods: microbiota and beyond. Curr Opin Biotechnol 2017; 44: 94-102.

11. Lavefve L, Marasini D, Carbonero F. Microbial ecology of fermented vegetables and non-alcoholic drinks and current knowledge on theirimpact on human health. In: Advances in food and nutrition research. Toldra F (ed.). Academic Press, Fayetteville, AR 2019; 147-185.

12. Dimidi E, Cox SR, Rossi M, Whelan K. Fermented foods: definitions and characteristics, impact on the gut microbiota and effects on gastrointestinal health and disease. Nutrients 2019; 11. pii: E1806.

13. Zabat M, Sano W, Wurster J, et al. Microbial community analysis of sauerkraut fermentation reveals a stable and rapidly established community. Foods 2018; 7. pii: E77.

14. Joyce OT, Chakraborty SK, Tripathi MK, et al. Quality characteristics of sauerkraut fermented by using a Lactobacillus paracasei starter culture grown in tofu whey. Food Sci Technol Int 2018; 24: 187-197.

15. Yu Z, Zhang X, Li S, et al. Evaluation of probiotic properties of Lactobacillus plantarum strains isolated from Chinese sauerkraut. World J Microbiol Biotechnol 2013; 29: 489-498.

16. Nielsen ES, Garnås E, Jensen KJ, et al. Lacto-fermented sauerkraut improves symptoms in IBS patients independent of product pasteurisation - a pilot study. Food Funct 2018; 9: 5323-5335.

17. Park KY, Jeong JK, Lee YE, Daily JW. Health benefits of kimchi (Korean fermented vegetables) as a probiotic food. J Med Food 2014; 17: 6-20.

18. Patra JK, Das G, Paramithiotis S, Shin HS. Kimchi and other widely consumed traditional fermented foods of Korea: a review. Front Microbiol 2016; 7: 1493.

19. Son SH, Jeon HL, Yang SJ, et al. In vitro characterization of Lactobacillus brevis KU15006, an isolate from kimchi, reveals anti-adhesion activity against foodborne pathogens and antidiabetic properties. Microb Pathog 2017; 112: 135-141.

20. Kim HJ, Noh JS, Song YO. Beneficial effects of kimchi, a Korean fermented vegetable food, on pathophysiological factors related to atherosclerosis. J Med Food 2018; 21: 127-135.

21. Alan Y. Culture fermentation of Lactobacillus in traditional pickled gherkins: Microbial development, chemical, biogenic amine and metabolite analysis. J Food Sci Technol 2019; 56: 3930-3939.

22. Zielińska D, Rzepkowska A, Radawska A, Zieliński K. In vitro screening of selected probiotic properties of Lactobacillus strains isolated from traditional fermented cabbage and cucumber. Curr Microbiol 2015; 70: 183-194.

23. Anandharaj M, Sivasankari B, Santhanakaruppu R, et al. Determining the probiotic potential of cholesterol-reducing Lactobacillus and Weissella strains isolated from gherkins (fermented cucumber) and south Indian fermented koozh. Res Microbiol 2015; 166: 428-439.

24. Liu YW, Liong MT, Tsai YC. New perspectives of Lactobacillus plantarum as a probiotic: The gut-heart-brain axis. J Microbiol 2018; 56: 601-613.

25. Jayachandran M, Xu B. An insight into the health benefits of fermented soy products. Food Chem 2019; 271: 362-371.

26. Niamah AK, Sahi AA, Al-Sharifi ASN. Effect of feeding soy milk fermented by probiotic bacteria on some blood criteria and weight of experimental animals. Probiotics Antimicrob Proteins 2017; 9: 284-291.
27. Cao ZH, Green-Johnson JM, Buckley ND, Lin QY. Bioactivity of soy-based fermented foods: A review. Biotechnol Adv 2019; 37: 223-238.

28. Aboderin Fl, Oyetayo O. Haematological studies of rats fed different doses of probiotic, Lactobacillus plantarum, isolated from fermenting corn slurry. Pak J Nutr 2006; 5: 102-105.

29. Cheng MC, Tsai TY, Pan TM. Anti-obesity activity of the water extract of Lactobacillus paracasei subsp. paracasei NTU 101 fermented soy milk products. Food Funct 2015; 6: 3522-3530.

30. Soka S, Suwanto A, Sajuthi D, Rusmana I. Impact of tempeh supplementation on gut microbiota composition in Sprague-Dawley rats. Res J Microbiol 2014; 9: 189-198.

31. Kapp JM, Sumner W. Kombucha: a systematic review of the empirical evidence of human health benefit. Ann Epidemiol 2019; 30: 66-70.

32. Teoh AL, Heard G, Cox J. Yeast ecology of Kombucha fermentation. Int J Food Microbiol 2004; 95: 119-126.

33. Coton M, Pawtowski A, Taminiau B, et al. Unraveling microbial ecology of industrial-scale Kombucha fermentations by metabarcoding and culture-based methods. FEMS Microbiol Ecol 2017; 93. doi: 10.1093/femsec/fix048. 